\title{
Erythrocyte as a link between basic and clinical research
}

\author{
C. Saldanha ${ }^{\mathrm{a}, \mathrm{b}}$ and J.P. Lopes de Almeida ${ }^{\mathrm{a}, \mathrm{b}, *}$ \\ ${ }^{a}$ University of Lisbon Medical School, Santa Maria Hospital, Lisbon, Portugal \\ ${ }^{\mathrm{b}}$ Institute of Biochemistry, Institute of Molecular Medicine, Faculty of Medicine, University of Lisbon, \\ Lisbon, Portugal
}

\begin{abstract}
We review the major hemorheological experimental studies that show the erythrocyte aggregation as a link between basic and clinical research. The results of the clinical cross-sectional and longitudinal studies presented here will highlight the possible association between erythrocyte aggregation and plasma fibrinogen. Basic studies conducted in vitro are also mentioned as for its relevance in answering questions raised in clinical settings, as well as and in understanding the underlying influent factors in the erythrocyte tendency to aggregate and disaggregate.
\end{abstract}

\section{Introduction}

In blood circulation the erythrocyte has the mission to furnish the tissues with oxygen and nitric oxide and receive the carbon dioxide in return [70]. To attain the gas exchange, at the microcirculation network, it is necessary to hold a proper blood flow cardiac output, vessel wall integrity, regulation of blood flow in the microvascular architecture network and normal blood viscosity [7, 30, 42, 53]. The tendency of red blood cells (RBCs) to aggregate and disaggregate is an influent factor in the rheological blood flow viscosity behaviour [9].

The increase of erythrocyte aggregation intensifies the microvascular flow resistance, fills capillaries (become with no flow) and at lower shear rates like in the venous circulation, the aggregation of red blood cells affects the blood velocities profile and increases blood viscosity [9, 34, 45]. Biomolecular, mechanical and physical factors influence RBCs aggregation and can be divided into: (i) extrinsic factors including the levels of plasma proteins (e.g. fibrinogen, lipoproteins, macroglobulins or immunoglobulins), hematocrit, particle size, plasma osmolality (particle number) and shear rate; and (ii) intrinsic factors e.g. RBCs shape, deformability and membrane surface properties [28, 48, 56, 69]. RBC surface properties and structure, such as surface charge and the ability of macromolecules to penetrate the membrane glycocalyx, greatly affect aggregation for cells suspended in a defined medium [18, 47].

\footnotetext{
${ }^{*}$ Corresponding author: J.P. Lopes de Almeida, Institute of Biochemistry, Institute of Molecular Medicine, Faculty of Medicine University of Lisbon Av. Prof. Egas Moniz, 1649-028 Lisbon, Portugal. E-mail: carlotasaldanha@fm.ul.pt.
} 
In the next sections we review some human pathological situations where increased levels of erythrocyte aggregation were found and also a few studies developed in vitro for the manipulation of the RBCs tendency to aggregate.

\subsection{Cross-sectional clinical studies}

The clinical studies trying to evaluate hemorheological parameters and discover their correlations with patient symptoms, lipid profile, hemodynamic, haemostatics and inflammatory factors, started in the last century.

We have verified that in a heterogeneous group of glaucoma patients, predominantly with chronic open angle, the erythrocyte aggregation is augmented [55]. Similar results were pointed by others later, in patients with normal tension glaucoma $[17,74]$. The great tendency of the red blood cells to aggregate allied to other hemorheological dysfunctions favour the developing of abnormalities at the microcirculation levels and damage of the optic nerve and consequently worsening the ocular blood flow [17, 74].

In a group of patients with chronic renal failure being submitted to chronic haemodialysis the erythrocyte aggregation index do not show significant difference when compared with the control group matched in age and gender, in spite of the increase fibrinogen levels observed in patients [6]. Apparently in this study the fibrinogen effect on erythrocyte aggregation tendency is surpassed by other influent factors mentioned above or by the anemia that favours the absence of changes in this red blood cell rheological property. The same apparent lack of influence was obtained in a group of renal transplant recipients where fibrinogen levels were similar to the control group but the erythrocyte aggregation has higher values in the recipients [4].

Other authors also verified an increase of RBCs aggregation in a group of renal transplant patients [20]. The aetiology of chronic renal failure is diverse and, consequently, when patients are submitted to kidney transplantation they do not sustain a homogeneous hemorheological behaviour. The recipients between them exhibited dissimilarities allied to the ocurrence of self immune system adaptation and inflammatory response [16, 21].

In a group of hypertensive patients with eye vascular dysfunctions, that were admitted to hospital, we have observed an increase in erythrocyte aggregation and of plasma fibrinogen concentrations [11]. The RBCs hyperaggregation obtained by us in these patients may be an underlying present factor in the reduction of peripheral tissue oxygenation verified by other authors in severe hypertensive patients [19]. We will come back to the results of our study [11] below on the topic of longitudinal studies.

In general, in hypertension several structural dysfunctions can occur like heart hypertrophy, fibrosis as well as at microcirculation where elevation of artery stiffness, impairment of artery remodelling and microvascular rarefaction may be present at different degrees of severity [10]. It was verified by Jung [31] that patients with long term essential hypertension when evaluated by intravital microscopy and the intramuscular by $\mathrm{pO} 2$ needle electrode, some present with primary microcirculatory disorders and others secondary disorders. In a previous work with patients with essential hypertension and renal dysfunction Jung [32] verified that when the number of capillaries per millimetre of epidermis are increased, the degree of retinal vascular changes (fundus hypertonicus) are more severe. Erythrocyte aggregation and plasma viscosity were elevated in those patients being the degree of retinal vascular alteration associated with plasma viscosity [32]. Changes in myogenic, metabolic, hormonal and trophic regulatory mechanisms present in vessel walls occur especially in those high perfused organs with low vascular resistance such as kidney, heart and brain. In general, aged humans have elevation of blood pressure, increased plasma 
fibrinogen, elevated platelet aggregability and augmented blood viscosity [14, 35, 38]. In spite of these evidences we conducted a hemorheological and cardiological study with normontensives elderly people [49]. This group presents both erythrocyte aggregation and fibrinogen values lower than younger age matched individuals without statistical significance [49]. The elderly group has spent a healthy happy past life with regular and moderate exercise and balanced intake diet [49].

In women with pregnancy-induced hypertension it was verified an increased RBCs aggregation and adhesiveness despite the absence of incremented fibrinogen level [25].

The authors in this work did not find any association between those hemorheologycal variables as opposed to other authors who reported that in a longitudinal study conducted with normal pregnant women both parameters increase and are associated [30]. Hemorheological study reported in women with preeclampsia reveal increased erythrocyte aggregation [72] but after therapeutic submission the high RBCs tendency to aggregate shows a reduction with labetalol and regression to the normal values with nifedipine [73].

The impairment of blood flow in macrocirculation caused by arteriosclerosis is a major determinant of microcirculation perfusion in coronary artery disease (CAD). Groups of patients with CAD with diagnostic of unstable or stable angina pectoris or with chest pain at hospital admission, before submission to fibrinolytic or any medical intervention, were studied [52]. In these groups of patients it was reported an increase in erythrocyte aggregation tendency and enhanced plasma levels of C-reactive protein and fibrinogen. The three parameters are considered indicators of cardiovascular disease [52]. The authors propose that increased RBCs aggregation seems unlike to contribute to the emergence of AMI [52]. The study conducted by Liuni [39] with healthy human volunteers in which ischemia/reperfusion injury was performed, has proved it does induce cardiovascular protection resulting from the administration of a HMG-CoA reductase inhibitor.

In the Aachen study three groups of patients namely with peripheral occlusive arterial disease, coronary artery disease and cerebrovascular insufficiency were evaluated and the results showed no differences among them in respect to the erythrocyte aggregation tendency and plasma fibrinogen concentration [37]. Actually, these values are higher when compared with those obtained in the healthy control group [37].

In the TAMCIS cross-sectional study a strong association was evidenced between the atherothrombotic risk factors and the degree of erythrocyte aggregation [3]. Other well important finding is the clear association between plasma fibrinogen levels and erythrocyte aggregation tendency at low grade inflammation manifested by the patients, whereas where serum lipids influence was only a minor determinant $[3,24$, 57, 77]. Another study in patients with atherothrombotic risk factors evidenced differences in gender profile having women significant higher values of erythrocyte aggregation than man with matched age [76].

The association between the strength of large RBCs aggregates and the inflammatory status and the high plasma fibrinogen concentration was also observed in patients with inflammatory bowel disease where a detrimental microcirculatory blood flow is present in the intestinal microvasculature [41].

\subsection{Longitudinal clinical studies}

In cross-sectional studies any biomolecule, cell property or cell function may not be considered a cause or a consequence for a disease neither named like a prognostic marker of further pathological events to occur in patients.

Beyond the rheological cross-longitudinal approach, follow-up studies have been developed and herein we will mention some of them. 
The above referred study [11] conducted in hypertensive patients with vascular dysfunctions at the ocular level, after admission to hospital, were followed during 45 days. At hospital discharge patients showed normal tensional levels, regression of the ophthalmological vasoconstriction to the normal values and improvement of the electrocardiographic dysfunction. However, the hemorheological parameters plasma viscosity and hematocrit decreased while the erythrocytes maintained the high tendency to aggregate which may be related to the intrinsic RBCs properties reported in hypertensive patients $[12,19,23,36$, $44,58]$.

A hemorheological clinical follow-up project of the Portuguese Society of Hemorheology and Microcirculation in association with the Lisbon Medical School (Biochemistry Institute and the Cardiology Department, UTIC-AC) was established and survivors of acute transmural myocardial infarctions were consecutively enrolled from 1994 to 1999, with clinical and cardiological evaluation and hemostatic, inflammatory and hemorheological parameters assessment at 6, 12, 24, 36 and 60 months [61, 63, 66-68].

During this period of time, the survivors were divided into two groups of patients according to the occurrence of cardiovascular events. At hospital discharge those patients that presented higher leukocytes count, higher leukocyte elastase enzyme activity, higher PAI-1, higher erythrocyte membrane viscosity, lower protein $\mathrm{C}$ activity or lower erythrocyte aggregation tendency, were found to develop cardiovascular events [61, 63, 66-68]. The absence of an association between erythrocyte aggregation and fibrinogen verified at hospital discharge appeared at 6 and at 12 months in an inverse relationship. The same behaviour was obtained between PAI-1 and fibrinogen and between PAI- 1 and plasma viscosity. The decrease of erythrocyte aggregation tendency determined at hospital discharge may result from the membrane rigidity determined in these patients [61]. The association observed between erythrocyte aggregation and inflammatory response (leukocyte elastase activity) and with hemostatic parameter (PAI-1) may explain the thrombotic events that occurred in the survivors. It is known that the presence of erythrocyte aggregates slow down the blood flow, reduce the level of capillary density and increase resistance to blood flow $[9,33$, 75]. Until 60 months of follow-up some haemostatic, hemorheological and inflammatory disturbances, at hospital discharge, seem to be long-term independent predictors of recurrent cardiovascular events in transmural myocardial infarction survivors [61, 63, 66-68].

Others have observed different patterns of erythrocyte aggregates mainly following the onset of pain prior to the event of infarction and advertise not to delay the medical intervention in these patients [71].

From the "Sicilian Study on Juvenile Myocardial Infarction", Caimi [13] presented the hemorheological results obtained from young adults before and after three months of acute myocardial infarction. The erythrocyte aggregation tendency did not show any variation between the two AMI stages neither between the patients assigned to groups with less of 2 or more than 2 risk factors, as well as between the groups assigned to 1 vessel or 2-3 vessels disease. In a case control study [78] of young patients with AMI aged less than forty five years old, subjects were submitted to the evaluation of hemorheological parameters and lipid profile between the third month and the first year after the occurrence of the event. No differences in the hemorheological parameters were verified between patients with differences on AMI type, on number and scores of stenosed vessels. In all patients erythrocyte aggregation, fibrinogen and triglycerides presented higher values than the control group, and the two last parameters, after multivariate adjustment were independently associated with the erythrocyte aggregation [5].

The anemia exhibited by patients with chronic renal failure on regular haemodyalysis was followed before and after three months of treatment with recombinant human erythropoietin [43]. A significant increase in the haemoglobin values was obtained despite no variations were verified in the RBCs aggregation [43]. 
A group of fourteen patients with familial hypercholesterolemia were treated with lovastatin for three months and the RBCs aggregation, and phospholipid membrane and lipid profile determined. After the intake of lovastatin there was a reduction in the plasma concentration of cholesterol and LDL cholesterol and this change correlates with a significant decrease in the membrane cholesterol/phospholipid ratio of the red blood cell membrane. The variation in the lipid composition verified is statistically related to the decrease in erythrocyte aggregability assessed [54].

From cross-sectional and longitudinal studies erythrocyte aggregation is (i) considered an independent risk factor for AMI, increasing over time in transmural myocardial survivors, (ii) evidenced as an independent predictor of recurrent cardiovascular events $[67,68,78]$, and (iii) associated with inflammation response [41, 52].

Fibrinogen is also present at high levels in plasma of inflammatory diseases, thereby being considered a acute phase protein [8] and demonstrated by Lominadze as a pro-inflammatory factor responsible for vascular dysfunction [40].

\subsection{Basic "in vitro" studies}

From the clinical settings there is no evidenced for a consistently direct or indirect relationship between the profile values of erythrocyte aggregation and fibrinogen. The polymorphism of the $\beta$-fibrinogen gene observed in healthy individuals is associated with differences is gender propensity to erythrocyte aggregation [8]. Others have suggested that in addition to fibrinogen, C-reactive protein may be an influent factor in enhancing the erythrocyte aggregation which present in inflammation and cardiovascular disease [64]. The authors propose that it must be (i) differentiated the contribution between the plasmatic and cellular factors and (ii) provided and determined the size of aggregates and the strength of the intercellular interaction [2]. Falcó attributed to erythrocyte aggregation apparatus used for its determination, the absence of its association with plasma fibrinogen concentration higher than $400 \mathrm{mg} / \mathrm{dL}$ [22].

We will present results obtained from in vitro studies that bring insights for the influence of fibrinogen on erythrocyte aggregation tendency with or without biological compounds in blood of healthy individuals and patients. Our approach was based on the dependence of the RBCs aggregation from their own properties and from the plasma composition.

We have verified in blood samples taken from healthy men that when enriched with fibrinogen the erythrocyte aggregation tendency increases [64]. The same behaviour was verified in the presence of oubain (a Na+, $\mathrm{K}^{+}$ATPase inhibitor) relatively to the oubain-and-fibrinogen -free control, as well as when compared with the fibrinogen-free control. There was no statistically significant difference in erythrocyte aggregation in aliquots with fibrinogen in absence or presence of oubain. From this study we may conclude that the increasing fibrinogen concentration are related to an increase in erythrocyte aggregation, thereby seeming not to be connected with the erythrocyte oubain-sensitive $\mathrm{Na}^{+}, \mathrm{K}^{+}$ATPase [26].

Pursuing our approach to study the erythrocyte membrane proteins, we focused on band 3 protein phosphorylation degree which was manipulated by using inhibitors of protein tyrosine kinase and protein tyrosine phosphatase [62]. Using blood samples from healthy individuals with plasma fibrinogen at normal range, the RBCs aggregation was found to be higher when band 3 is phosphorylated than when dephosphorylated besides both band 3 states have lower values than the control aliquot. The band 3 protein phosphorylation status becomes an intrinsic influent factor in this erythrocyte rheological property [62]. 
The stimulation of the erythrocyte redox status by adding dithiothreitol in blood samples taken from healthy humans with normal values of plasma fibrinogen, revealed no changes in RBCs aggregation [1].

The increase in nitric oxide (NO) efflux and mobilization occurred in RBCs when in presence of acetylcholine and the enhance RBCs NO influx occur by the effect of Spermine NONOate [48]. This gas movement induces respectively a decrease or maintenance of RBCs aggregation tendency in blood samples of healthy donors with normal values of plasma fibrinogen [48]. A signal transduction mechanism that includes Gi protein to explain increase erythrocyte tendency under effects of acetylcholine or stimuli on Gi protein itself was pointed by us and Moravyov et al. $[15,50]$. The last group of authors shows that stimulating the calcium influx and the adenylyl cyclase -cAMP system increases the aggregation of the RBCs [51]. Blood samples were obtained from women undergoing transdermic 17 $\beta$-estradiol (50 $\mu \mathrm{g} /$ diarly). From incubations of blood aliquots with $17 \beta$-estradiol $10^{-5} \mathrm{M}$, two sub-groups with high and low RBCs membrane fluidity were obtained which showed increase osmolality and decreased erythrocyte aggregation [27]. Some unknown molecular effect nullifies the direct positive effect of increased osmolality on the RBCs tendency to aggregate [59]. We verified by fluorescence spectroscopy the alteration on fibrinogen molecule conformation by binding of $17 \beta$-estradiol with high affinity (association constant $=6.47 \times 10^{6} \mathrm{M}^{-1}$ ) [60]. Other molecular interactions may occur between therapeutically circulating plasma molecules and fibrinogen that justify diverse kinds of association or lack of them between fibrinogen levels and RBCs aggregation tendency. For example we studied a group of hypercholestreolemic patients submitted during three months to lovastatin therapy that does not show any changes in the erythrocyte aggregation tendency [65]. However when blood samples taken before treatment onset where incubated with different lovastatin concentration a decrease of the RBCs aggregation tendency was observed [65].

Beyond the effects of blood mechanical factors, plasma substances and intrinsic erythrocyte properties influence on RBCs aggregation in vivo, or in vitro others factors must be taken into account. So, there must be considered for example (i) different apparatus for its measurement and (ii) the concepts of RBCs aggregation and RBCs aggregability defined by Meiselman who dedicates great part of his research (more than 45 years) in the field of erythrocyte aggregation [46].

$\mathrm{Al}$ the studies reported in this review was referred to erythrocyte aggregation.

\section{Acknowledgments}

The authors are grateful to Emilia Alves for typewrite the manuscript. This work was supported by "Fundação para a Ciência e Tecnologia".

\section{References}

[1] J.P. Almeida, F.A. Carvalho, T. Freitas and C. Saldanha, Modulation of hemorheological parameters by the erythrocyte redox thiol status, Clin Hemorheol Microcirc 40 (2008), 99-11.

[2] R.B. Ami, G. Barshtein, D. Zeltser, Y. Goldberg, I. Shapia, A. Roth, G. Keren, H. Miller, V. Prochorov, A. Eldor, S. Berliner and S. Yedgar, Parameters of red blood cell aggregation as correlates of the inflammatory state, Am J Physiol Heart Circ Physiol 280 (2001) H1982-H1988.

[3] E.B. Assayag, N. Bornstein, I. Shapira, T. Mardi, Y. Goldin, T. Tolshinski, Y. Vered, V. Zakuth, M. Burke, S. Berliner and D.S. Bonet, Inflammation-sensitive proteins and erythrocyte aggregation in atherothrombosis, Int J Cardiol $\mathbf{9 8}$ (2005), $271-276$. 
[4] J. Barbas, L. Cardoso, Y. Pinto, C. Saldanha and J. Martins e Silva, Blood rheological observation in renal transplant patients, Rev Port Hemorreol 4 (1990), 207-213.

[5] J. Barbas, M. Martins-Prata, L. Cardoso, F. Teixeira Sousa, C. Moreira, J. Pintos Santos, C. Saldanha and J. Martins e Silva, Effects of partial correction of anaemia with recombinant human erythropoietin on the haemorheological profile of haemodialysis patients, Rev Port Hemorreol 3 (1989), 269-276.

[6] J. Barbas, C. Saldanha, C. Moreira, L. Cardoso, A. Santos, T. Quintão and J. Martins e Silva, Determination of some haemorheologic parameters in patients with chronic renal failure being submitted to chronic haemodialysis, Rev Port Hemorreol 2 (1988), 145-152.

[7] O.K. Baskurt and H.J. Meiselman, Blood rheology and hemodynamics, Semin Thromb Hemost 29 (2003), 435-450.

[8] E. Ben Assayag, I. Bova, S. Berliner, H. Peretz, S. Usher, I. Shapira and N.M. Bornstein, Gender differences in the expression of erythrocyte aggregation in relation to B beta-fibrinogen gene polymorphisms in apparently healthy individuals, Thromb Haemost 95 (2006), 428-433.

[9] J.J. Bishop, P.R. Nance, A.S. Popel, M. Intaglietta and P.C. Johnson, Relationship between erythrocyte aggregation size and flow rate in skeletal muscle venules, Am J Physiol Heart Circ Physiol 286 (2004), H113- H120.

[10] H.A.J.S. Boudier, G.M.S. Cohuet, M. Baumann and M.E. Safar, The heart macrocirculation and microcirculation in hypertension: A unifying hypothesis, Hyperten Suppl 21 (2003), S19-S23.

[11] J. Braz-Nogueira, A. Freitas, C. Moreira, C. Saldanha, J. Martins e Silva, P. Souza-Ramalho and J. Nogueira da Costa, Hipertensão em fase acelerada, Estudo cardiológico, oftalmológico e hemorreológico, Rev Port Cardiol 7 (1988), 305-318.

[12] G. Caimi, Erythrocyte platelet and polymorphonuclear leukocyte membrane dynamic properties in essential hypertension, Clin Hemorheol Microc 17 (1997), 100-208.

[13] G. Caimi, E. Hoffmann, M. Montana, B. Canino, F. Dispensa, A. Catania and R. Lo Presti, Haemorheological pattern in young adults with acute myocardial infarction, Clin Hemorheol Microcirc 29 (2003), 11-18.

[14] C. Carallo, C. Irace, M.S. De Franceschi, F. Coppoletta, R. Tiriolo, C. Scicchitano, F. Scavelli and A. Gnasso, The effect of aging on blood and plasma viscosity. An 11.6 years follow-up study, Clin Hemorheol Microcirc 47 (2011), 67-74.

[15] F.A. Carvalho, J.P. Lopes de Almeida, T. Freitas-Santos and C. Saldanha, Modulation of erythrocytes acetylcholinesterase activity and its association with G protein. Band 3 interactions, J Memb Biol 228 (2009), 89-97.

[16] F.A. Carvalho, A.V. Maria, J.M. Braz Nogueira, J. Guerra, J. Martins-Silva and C. Saldanha, The relation between the erythrocyte nitric oxide and hemorheological parameters, Clin Hemorheol Microcirc 35 (2006), 341-347.

[17] H.C. Cheng, C.M. Chan, S.I. Yeh, J.H. Yu and D.Z. Liu, The hemorheological mechanisms in normal tension glaucoma, Curr Eye Res 36 (2011), 647-653.

[18] S. Chien and L.A. Lang, Physicochemical basis and clinical implications of red cell aggregation, Clin Hemorheol 7 (1987), 71-91.

[19] G. Cicco, P. Vicenti, G.D. Stingi, Tarallo and A. Pirrelli, Hemorheology in complicated hypertension, Clin Hemorheol Microcirc 21 (1999), 315-319.

[20] K. Damman, P.R. Kalra and H. Hillege, Pathophysiological mechanisms contributing to renal dysfunction in chronic heart failure, J Ren Care 36(Suppl 1) (2010), 18-26.

[21] P. De Paolis, A. Favarò, A. Piola, F. Martini, G. Cristiana, C. Agrati, M. Iappelli, S. Di. Giulio, "Immuknow" to measurement of cell-mediated immunity in renal transplant recipients undergoing short-term evaluation, Transplant Proc 43 (2011), 1013-1016.

[22] C. Falcó, A. Vayá, M. Simó, T. Contreras, M. Santaolaria and J. Aznar, Influence of fibrinogen levels on erythrocyte aggregation determined with the Myrenne aggregometer and the Sefam erythro-aggregometer, Clin Hemorheol Microcirc 33 (2005), 145-151.

[23] P. Foresto, M. D’ Arrigo, L. Racca, F. Filippini, R. Gallo, J. Valverde and R.J. Rasia, Comparative analysis of aggregate shapes by digitized microscopic images, Application to hypertension, Clin Hemorheol Microcirc 26 (2002), 137-144.

[24] R. Fusman, R. Rotstein, S. Berliner, K. Elishkewich, A. Rubinstein, E. Izkhacov, D. Zeltser, D. Avitzour, N. Arber and I. Shapira, The concomitant appearance of aggregated erythrocytes, leukocytes and platelets in the peripheral blood of patients with risk factors for atherothrombosis, Clin Hemorheol Microcirc 25 (2001), 165-173.

[25] R. Gamzu, R. Rotstein, R. Fusman, D. Zeltser, A.S. Berliner and M.J. Kupferminc, Increased erythrocyte adhesiveness and aggregation in peripheral venous blood of women with pregnancy-induced hypertension, Obstet Gynecol $\mathbf{9 8}$ (2001), 307-312.

[26] I. Gonçalves, R. Mesquita, C. Saldanha and J. Martins e Silva, Oubain role on erythrocyte/fibrinogen interaction, Blood Coagulation and Fibrinolysis 10 (1999), S107 
[27] I. Gonçalves, C. Saldanha and J. Martins e Silva, $\beta$-estradiol effect on erythrocyte aggregation-a controlled in vitro study, Clin Hemorheol Microcirc 25 (2001), 127-134.

[28] S. Hilário, C. Saldanha and J.Martins e Silva, An in vitro study of adrenaline effect on human erythrocyte properties in both genders, Clin Hemorheol Microcirc 28 (2003), 89-98.

[29] A. Huisman, J.G. Aarnoudse, M. Krans, H.J. Huijse, V. Fidler and W.G. Zijlstra, Red cell aggregation during normal pregnancy, Br J Haematol 69 (1988), 121-124.

[30] F. Jung, From hemorheology to microcirculation and regenerative medicine, Fåhraeus Lecture 2009, Clin Hemorheol Microcirc 45 (2010), 79-99.

[31] F. Jung, W. Kolepke, S. Spitzer, H. Kiesewetter, K.W. Ruprecht, R. Bach, H. Schieffer and E. Wenzel, Primary and secondary microcirculatory disorders in essential hypertension, Clin Investig 71 (1993), 132-138.

[32] F. Jung, S. Spitzer, H. Kiesewetter, M. Feldmann, G. Kotitschke, C. Blum, E. Wenzel and G.A. Jutzler, Comparative investigation of the microcirculation in patients with hypertension and healthy adults, Klin Wochenschr 64 (1986), 956-961.

[33] S. Kim, A.S. Popel, M. Intaglieta and P.C. Johnson, Effect of erythrocyte aggregation at normal human level on functional capillary density in rats spinotrapezius muscle, AM J Physiol Heart Circ Physiol 290 (2006), H941-H520.

[34] S. Kim, A.S. Popel, M. Intaglietta and P.C. Johnson, Aggregate formation of erythrocytes in potcapillary venules, Am J Physiol heart Circ Physiol 288 (2005), 584.

[35] K. Koltai, G. Feher, P. Kenyeres, I. Lenart, T. Alexy, B. Horvath, Z. Marton, G. Kesmarky and K. Toth, Relation of platelet aggregation and fibrinogen levels to advancing age in aspirin- and thienopyridine-treated patients, Clin Hemorheol Microcirc 40 (2008), 295-302.

[36] E. Konstantinova, L. Ivanova, T. Tolstaya and E. Mironova, Rheological properties of blood and parameters of platelets aggregation in arterial hypertension, Clin Hemorheol Microcirc 35 (2006), 135-138.

[37] J. Koscielny, E.M. Jung, C. Mrowietz, H. Kiesewetter and R. Latza, Blood fluidity, fibrinogen, and cardiovascular risk factors of occlusive arterial disease: Results of the Aachen study, Clin Hemorheol Microcirc 31 (2004), 185-195.

[38] A. Kovács, Z. Szikszai, E.Várady and S. Imre, Study on the hemorheological parameters of oldest-old residents in the East-Hungarian city, Debrecen, Clin Hemorheol Microcirc 35 (2006), 83-88.

[39] A. Liuni, M.C. Luca, T. Gori and J.D. Parker, The endothelial-protective effects of HMG-CoA reductase inhibition in the setting of ischemia and reperfusion injury, Clin Hemorheol Microcirc 45 (2010), 161-167.

[40] D. Lominadze, W.L. Dean, S.C. Tyagi and A.M. Roberts, Mechanisms of fibrinogen-induced microvascular dysfunction during cardiovascular disease, Acta Physiol 198 (2010), 1-13.

[41] N. Maharshak, Y. Arbel, I. Shapira, S. Berliner, R. Ben-Ami, S. Yedgar, G. Barshtein and I. Dotan, Increased strength of erythrocyte aggregates in blood of patients with inflammatory bowel disease, Inflamm Bowel Dis 15 (2009), 707-713.

[42] A. Marossy, P. Svorc, I. Kron and S. Gresová, Hemorheology and circulation, Clin Hemorheol Microcirc 42 (2009), 239-258.

[43] M. Martinez, A. Vayá, R. Marti, L. Gil, I. Lluch, R. Carmena and J. Aznar, Erythrocyte membrane choleterol/phospholipid and hemorheological modifications in familiar hypercholesterolemia trated with lovastatin, Thromb Res 83 (1996), 375-388.

[44] J. Martins e Silva, M. Carlota Proença, J. Braz Nogueira, J. Gorjão-Clara, J. Nogueira da Costa and C. Manso, Erythrocyte actylcholinesterase in essential hypertension, J Neural Transm 49 (1980), 127-132.

[45] G. Mchedlishvili, L. Gobejis and N. Beritashvili, Effect of intensified red blood cell aggregability on arterial pressure and mesenteric microcirculation, Microvas Res 45 (1993), 233-242.

[46] H.J. Meiselman, Red blood cell aggregation: 45 years being curious. Poieseuille Medal Award Lecture 2008, Biorheology 46 (2009), 1-19.

[47] H.J. Meiselman, Red blood cell role in RBC aggregation: 1963-1993 and beyond, Clin Hemorheology 13 (1993), $575-592$.

[48] R. Mesquita, I. Pires, C. Saldanha and J. Martins-Silva, Effects of acetylcholine and spermineNONOate on erythrocyte hemorheologic and oxygen carrying properties, Clin Hemorheol Microcirc 25 (2001),153-163.

[49] E. Mota, D. Alves da Silva, A. Ventura Espiga, C. Morais, R. Ranchhod, L. Cardoso, C. Saldanha, J. Martins e Silva and J. Nogueira da Costa, Estudo cardiológico e hemorreologico de normotensos idosos, Rev Port Cardiol 10 (1989), 365-371.

[50] A.V. Muravyov, I.A. Tikhomirova, A.A. Maimistova and S.V. Bulaeva, Extra- and intracellular signaling pathways under red blood cell aggregation and deformability changes, Clin Hemorheol Microcirc 43 (2009), 223-232.

[51] A.V. Muravyov, I.A. Tikhomirova, A.A. Maimistova, S.V. Bulaeva, A.V. Zamishlayev and E.A. Batalova, Crosstalk between adenylyl cyclase signaling pathway and $\mathrm{Ca} 2+$ regulatory mechanism under red blood cell microrheological changes, Clin Hemorheol Microcirc 45 (2010), 337-345.

[52] C. Pfafferott, G. Moessmer, A.M. Ehrly and R.M. Bauersachs, Involvement of erythrocyte aggregation and erythrocyte resistance to flow in acute coronary syndromes, Clin Hemorheol Microcirc 21 (1999), 35-43. 
[53] A.S. Popel and P.C. Johnson, Microcirculation and hemorheology, Annu Rev Fluid Mech 37 (2005), $43-69$.

[54] R. Ross, Atherosclerosis - an inflammatory disease, N Engl J Med 340 (1999), 115-126.

[55] P. Ramalho, C. Saldanha, L. Cardoso, D. Santos and J. Martins e Silva, Hemorheological changes in glaucoma, New Trends Ophtalmol 1 (1986), 45-51.

[56] M.W. Rampling, H.J. Meiselman, B. Neu and O.K. Baskurt, Influence of cell-specific factors on red blood cell aggregation, Biorheology 41 (2004), 91-12.

[57] O. Rogowski, D. Zeltser and S. Rotstein, Correlated of adhesive properties for both white and red blood cells during inflammation, Biorheology 37 (2000), 361-370.

[58] C. Saldanha, J. Braz-Nogueira, S. Marques, C. Moreira, J. Nogueira da Costa and J. Martins e Silva, Some abnormalities of red cell membrane in essential hypertension, Rev Port Hemorreol 1 (1987), 3-8.

[59] C. Saldanha and J. Martins e Silva, Effect of plasma osmolality on erythrocyte aggregation índex. Hémorheologie et aggregation erythrocytaire, vol 3 Théorie et applications cliniques. J.F. stoltz, M. Donner, Al Copley (eds), Editions Medicals Internationales, Paris, 1991, 142-145.

[60] C. Saldanha, C. Moreira and J. Martins e Silva, Role of lovastatin in hemorheological and biochemical parameters of erythrocyte from hypercholesterolemic patients, A follow-up study, Atherosclerosis 115 (1995), S98.

[61] C. Saldanha, Sargento, J. Monteiro, C. Perdigão and J. Martins e Silva, Impairment of the erythrocte membrane fluidity in survivors of acute myocardial infarction, Prospective study, Clin Hemorheol Microcir 20 (1999), 111-116.

[62] C. Saldanha, A.S. Silva, S. Gonçalves and J. Martins-Silva, Modulation of erythrocyte hemorheological properties by band 3 phosphorylation and dephosphorylation, Clin Hemorheol Microcirc 36 (2007), 183-194.

[63] L. Sargento, H.S. Do Rosário, C. Perdigão, J. Monteiro, C. Saldanha and J. Martins e Silva, Long-term prognostic value of the hemorheological profile in transmural myocardial infarction survivors: 60-month clinical follow-up, Rev Port Cardiol 21 (2002), 1263-1275.

[64] L. Sargento, C. Saldanha and J. Martins e Silva, Fibrinogen and the erythrocyte membrane: Na "in vitro" study, 3rd International Conference of Clinical Hemorheology, 10th International Congress of Biorheology 1999, Biorheology 36 (1999), 128.

[65] L. Sargento, C. Saldanha and J. Martins e Silva, Role of lovastatin in biochemical properties of erythrocyte from hypercholesterolemic patients, Atherosclerosis 115 (1995) S99.

[66] L. Sargento, C. Saldanha and J. Martins e Silva, Anomalias hemorreológicas em sobreviventes de enfarte agudo do miocárdio como marcadores de prognóstico, Boletim SPHM 13 (1998), 4-9.

[67] L. Sargento, C. Saldanha, J. Monteiro, C. Perdigão and J. Martins e Silva, Evidence of prolonged disturbances in the haemostatic, hemorheologic and inflammatory profiles in transmural myocardial infarction survivors. A 12-month followup study, J Thromb Haemost 89 (2003), 892-903.

[68] L. Sargento, C. Saldanha, J. Monteiro, C. Perdigão and J. Martins e Silva, Long-term prognostic value of protein C activity, erythrocyte aggregation and membrane fluidity in transmural myocardial infarction. 36-month follow-up, Thromb Haemost 94 (2005), 380-388.

[69] T. Shiga, N. Maeda and K. Kon, Erythrocyte rheology, Crit Rev Oncol Hematol 10 (1990), 9-48.

[70] P. Sonveaux, I.I. Lobysheva, O. Feron and T.J. McMahon, Transport and peripheral bioactivities of nitrogen oxides carried by red blood cell hemoglobin: Role in oxygen delivery, Physiology 22 (2007), 97-112.

[71] A. Steinvil, S. Berliner, I. Shapira, O. Rogowski, D. Justo, J. George, A. Halkin, G. Keren, A. Finkelstein, S. Banai and Y. Arbel, Time to rheology in acute myocardial infarction: Inflammation and erythrocyte aggregation as a consequence and not necessarily as precursors of the disease, Clin Res Cardiol 99 (2010), 651-656.

[72] S. Stoeff, S. Jovtchev, I. Dikov, D. Kolarov, T. Galabova, N. Trifonova, S. Hadjieva, A. Mladenova, L. Lambreva and M. Penev, Quantitative expediency assessment of the zeta sedimentation ratio and the plasma viscosity in arterial hypertension research, Clin Hemorheol Microcirc 39 (2008), 381-384.

[73] A.L. Tranquilli, G.G. Garzetti, G. De Tommaso, M. Boemi, E. Lucino, P. Fumelli and C. Romanini, Nephedipine treatment in preeclampsia reverts the increased erythrocyte aggregation to normal, Am J Obstet Gynecol 167 (1992), 942-945.

[74] M. Vetrugno, G. Cicco, F. Cantatore, L. Arnese, N. Delle Noci and C. Sborgia, Red blood cell deformability, aggregability and cytosolic calcium concentration in normal tension glaucoma, Clin Hemorheol Microcirc 31 (2004), 295-302.

[75] O. Yalcin, H.J. Meiselman, J.K. Amstrong and O.K. Baskurt, Effect of red blood cell aggregation on blood flow resistance in an isolated-perfused guinea pig pig heart preparation, Biorheology 42 (2005), 511520.

[76] D. Zeltser, O. Rogowski, S. Berliner, T. Mardi, D. Justo, J. Serov, M. Rozenblat, D. Avitzour and I. Shapira, Sex differences in the expression of haemorheological determinants in individuals with atherothrombotic risk factors and in apparently healthy people, Heart 90 (2004), 277-281. 
[77] D. Zeltser, J. Serov, T. Mardi, O. Rogowski, T. Tulshinski, Y. Goldin, D. Justo, S. Aharonov, M. Rozenblat, S. Berliner, I. Shapira and A. Rubinstein, Serum lipids as minor determinants of the degree of erythrocyte adhesiveness/aggregation in the peripheral blood of individuals with low grade inflammation and moderately increased serum lipids, Clin Hemorheol Microcirc 31 (2004), 161-167.

[78] E. Zorio, J. Murado, D. Arizo, J. Rueda, D. Corella, M. Simó and A. Vayá, Haemorheological parameters in young patients with acute myocardial infarction, Clin Clin Hemorheol Microcirc 39 (2008), 33-41 